\title{
A NEW PROOF OF THE BILINEAR T(1) THEOREM
}

\author{
JAROD HART
}

(Communicated by Alexander Iosevich)

\begin{abstract}
A new simple proof of the bilinear T(1) Theorem in the spirit of the proof of Coifman-Meyer of the celebrated result of David and Journé in the linear case is presented. This new proof is obtained independently of the linear $\mathrm{T}(1)$ Theorem by combining recent bilinear square function bounds and a paraproduct construction.
\end{abstract}

\section{INTRODUCTION}

A central theme of harmonic analysis over the past few decades has been the study of singular integral operator bounds in terms of subtle cancellation conditions. The most famous result in this study is the T(1) Theorem for linear CalderónZygmund operators, proved by David-Journé [7]:

$\mathbf{T}(\mathbf{1})$ Theorem. Let $T: \mathscr{S} \rightarrow \mathscr{S}^{\prime}$ be a linear singular integral operator with a standard Calderón-Zygmund kernel. Then $T$ can be extended to a bounded operator on $L^{2} \rightarrow L^{2}$ if and only if $T$ has the weak boundedness property and $T 1, T^{*} 1$ are in $B M O$.

In [7] the authors considered smooth truncations of a singular integral operator and worked in operator norm topologies using the Cotlar-Knapp-Stein Lemma to prove the almost orthogonality of these truncations. In the bilinear setting, this approach quickly fails. The Cotlar-Knapp-Stein Lemma requires one to define truncated operators that map a Hilbert space into itself. In the linear setting this is not a problem, and in fact is natural to work in the Hilbert space $L^{2}$. In the bilinear setting, the truncated operators map from a product of Lebesgue spaces into other Lebesgue spaces. This immediately disqualifies the use of the Cotlar-Knapp-Stein Lemma as it is used in [7.

There are other proofs of the $\mathrm{T}(1)$ Theorem. For example, the simple proof of Coifman and Meyer in [5] avoided the almost orthogonality lemma by using a different representation of the operator in terms of smooth ones and directly estimated the operator norm using square functions.

Christ-Journé [6] and Grafakos-Torres [15] obtained versions of the T(1) Theorem in a multilinear setting relying somehow on the linear result. We state an equivalent bilinear version that we will be considering in this work. See Section 2 for definitions.

Received by the editors May 25, 2012 and, in revised form, May 26, 2012; July 11, 2012; July 12, 2012; August 21, 2012; and October 3, 2012.

2010 Mathematics Subject Classification. Primary 42B20; Secondary 42B25.

Key words and phrases. Bilinear, T(1) Theorem, Calderón-Zygmund operators, square function.

The author was supported in part by NSF Grant \#DMS1069015. 
Theorem 1.1. Let $T: \mathscr{S} \times \mathscr{S} \rightarrow \mathscr{S}^{\prime}$ be a bilinear singular integral operator with standard kernel. Then $T$ can be extended to a bounded operator from $L^{p} \times L^{q}$ into $L^{r}$ for all $1<p, q<\infty$ with $\frac{1}{p}+\frac{1}{q}=\frac{1}{r}$ if and only if $T$ has the weak boundedness property and $T(1,1), T^{* 1}(1,1), T^{* 2}(1,1)$ are in $B M O$.

In [6], the authors used the conditions that $T$ satisfies a certain weak boundedness property (different from the one we will use in this work) and $T(1,1), T^{* 1}(1,1)$, $T^{* 2}(1,1) \in B M O$ to prove the result using an approach similar to the proof of Coifman-Meyer. They rely on almost orthogonality in a different form and Carleson measure estimates. In [14 the authors replaced the assumptions that $T$ is weakly bounded and $T^{* j}(1,1) \in B M O$ for $j=0,1,2$ with the single equivalent condition

$$
\sum_{j=0}^{2} \sup _{\xi, \eta \in \mathbb{R}^{n}}\left\|T^{* j}\left(e^{i \xi \cdot(\cdot)}, e^{i \eta \cdot(\cdot)}\right)\right\|_{B M O}<\infty .
$$

This is also a bilinear version of an equivalent condition in the linear case proved in [7. The proof in [15] followed an approach combining restricted boundedness properties as in the arguments in Stein's proof 21] of the T(1) Theorem and relying again on linear theory. We also point out that a version of a reduced bilinear $\mathrm{T}(1)$ Theorem in the context of Triebel-Lizorkin spaces was obtained by Bényi [1] (with yet another weak boundedness property) using smooth atomic and molecular decompositions as in the works of Meyer [20, Frazier-Han-Jawerth-Weiss [9], FrazierTorres-Weiss [10, Torres 22] and others in the linear case. A different bilinear $\mathrm{T}(1)$ Theorem for modulation invariant operators generalizing the bilinear Hilbert transform (and hence outside the multilinear Calderón-Zygmund theory) was more recently obtained by Bényi-Demeter-Nahmod-Thiele-Torres-Villarroya [2] .

As in 6, the proof we present here for the bilinear version of the $T(1)$ Theorem follows the Coifman-Meyer approach. The main difference in our method, however, is that we are able to take advantage of recent bilinear square functions from [16] not available before, which simplify some of the arguments. The bilinear square function estimates reduce matters to some very natural computations avoiding the use of the linear T(1) Theorem. We also construct bilinear paraproducts that allow us to further simplify the proof to the case of a reduced theorem.

All of the results in this work can be extended to the $m$-linear setting with obvious modifications of the proofs, but for simplicity we restrict ourselves to working in the bilinear case.

\section{FURTHER TECHNICAL DEFINITIONS AND BACKGROUND MATERIAL}

As usual, define the Schwartz space $\mathscr{S}$ to be the collection of rapidly decreasing, $C^{\infty}$ functions with the topology generated by the semi-norms

$$
\rho_{\alpha, \beta}(f)=\sup _{x \in \mathbb{R}^{n}}\left|x^{\alpha} \partial_{x}^{\beta} f(x)\right|
$$

for $\alpha, \beta \in \mathbb{N}_{0}^{n}$. We also define the Fourier transform of a function $f$ by

$$
\widehat{f}(\xi)=\int e^{-i x \xi} f(x) d x
$$

whenever the integral converges. We denote by $\mathcal{D} \subset \mathscr{S}$ the subspace of functions $f \in \mathscr{S}$ with compact support, and $\mathscr{S}_{0} \subset \mathscr{S}$ to be the subspace of functions $f \in \mathscr{S}$ with infinite vanishing moment, i.e. $\partial_{\xi}^{\alpha} \widehat{f}(0)=0$ for all $\alpha \in \mathbb{N}_{0}^{n}$. Given a 
function $f: \mathbb{R}^{n} \rightarrow \mathbb{C}$, we use the typical notation for $t>0, \varphi_{t}(x)=t^{-n} \varphi\left(t^{-1} x\right)$, $\varphi^{y}(x)=\varphi(x-y)$ and $\varphi^{y, t}(x)=\varphi\left(\frac{x-y}{t}\right)$.

Definition 2.1. Let $T: \mathscr{S} \times \mathscr{S} \rightarrow \mathscr{S}^{\prime}$ be a bilinear operator. Then $T$ is a bilinear singular integral operator with standard Calderón-Zygmund kernel if there exists a distribution $K \in(\mathscr{S} \times \mathscr{S} \times \mathscr{S})^{\prime}$ so that

$$
\langle T(f, g), h\rangle=\langle K, h \otimes f \otimes g\rangle
$$

for all $f, g, h \in \mathscr{S}$, where $K$ agrees with a locally integrable function $K(x, y, z)$ on $\mathbb{R}^{3 n} \backslash\left\{(x, x, x): x \in \mathbb{R}^{n}\right\}$, that is, for $\operatorname{supp}(f) \cap \operatorname{supp}(g) \cap \operatorname{supp}(h)=\emptyset$,

$$
\langle T(f, g), h\rangle=\iiint K(x, y, z) h(x) f(y) g(z) d x d y d z
$$

and $K$ satisfies

$$
\begin{aligned}
& |K(x, y, z)| \leq \frac{A}{(|x-y|+|x-z|)^{2 n}} \text { for } x \neq y, \text { or } x \neq z, \\
& \left|K(x, y, z)-K\left(x^{\prime}, y, z\right)\right| \leq \frac{A\left|x-x^{\prime}\right|^{\gamma}}{(|x-y|+|x-z|)^{2 n+\gamma}} \\
& \quad \text { for }\left|x-x^{\prime}\right| \leq \max (|x-y|,|x-z|) / 2, \\
& \left|K(x, y, z)-K\left(x, y^{\prime}, z\right)\right| \leq \frac{A\left|y-y^{\prime}\right|^{\gamma}}{(|x-y|+|x-z|)^{2 n+\gamma}} \\
& \left|K(x, y, z)-K\left(x, y, z^{\prime}\right)\right| \leq \frac{A\left|z-z^{\prime}\right|^{\gamma}}{(|x-y|+|x-z|)^{2 n+\gamma}} \\
& \text { for }\left|z-z^{\prime}\right| \leq \max (|x-y|,|x-z|) / 2
\end{aligned}
$$

for some $0<\gamma \leq 1$.

We wish to prove a boundedness criterion for bilinear singular integral operators using the weak boundedness property and conditions of the form $T(1,1)$. Although $T(1,1)$ is not defined a priori as $1 \notin \mathscr{S}$, we define $T(f, g)$ for $f, g \in C^{\infty} \cap L^{\infty}$ modulo a constant similar to the linear case.

Definition 2.2. Let $T: \mathscr{S} \times \mathscr{S} \rightarrow \mathscr{S}^{\prime}$ be a bilinear singular integral operator and $\eta \in \mathcal{D}$ with $\eta \geq 0$ and $\eta \equiv 1$ on a neighborhood of the origin. Define $\eta_{R}(x)=\eta(x / R)$ and

$$
T(f, g)=\lim _{R \rightarrow \infty} T\left(f \eta_{R}, g \eta_{R}\right)-c_{R}
$$

for $f, g \in C^{\infty} \cap L^{\infty}$, where this limit is taken in $\mathcal{D}^{\prime}$ and

$$
c_{R}=\iint_{|y|,|z|>1} K(0, y, z) \eta_{R}(y) f(y) \eta_{R}(z) g(z) d y d z .
$$

This limit exists in $\mathcal{D}^{\prime}$ and is consistent with $T$ modulo a constant just as in the linear case. So when computing $T(f, g)$ for $f, g \in C^{\infty} \cap L^{\infty}$, we must restrict ourselves to pairing $T\left(f \eta_{R}, g \eta_{R}\right)$ with functions $\phi \in \mathcal{D}$ where $\phi$ has integral zero to be consistent with the definition of $T$. For the details of these remarks, see for example [15].

We now define the weak boundedness property for linear and bilinear singular integral operators. We will not use the linear weak boundedness property in this 
work, but it is worth stating to compare with the bilinear weak boundedness property, as there have been a number of different definitions used. See for example the definitions of the bilinear weak boundedness property in [6] and [1].

Definition 2.3. A function $\phi \in \mathcal{D}$ is a normalized bump of order $M$ if $\operatorname{supp}(\phi) \subset$ $B(0,1)$ and for all $\alpha \in \mathbb{N}_{0}^{n}$ with $|\alpha| \leq M$,

$$
\left\|\partial^{\alpha} \phi\right\|_{L^{\infty}} \leq 1
$$

Let $T: \mathscr{S} \rightarrow \mathscr{S}^{\prime}$ be a linear singular integral operator. Then $T$ has the weak boundedness property for linear singular integral operators if there exists an $M \in$ $\mathbb{N}_{0}$ such that for all normalized bumps $\phi_{1}$ and $\phi_{2}$ of order $M, x, y \in \mathbb{R}^{n}$ and $t>0$,

$$
\left|\left\langle T \phi_{1}^{x, t}, \phi_{2}^{y, t}\right\rangle\right| \lesssim t^{n}
$$

We remark that typically in the literature the above $W B P$ is stated for the same translation $x=y$ of the smooth bumps. However, given the size estimate on the standard kernel, the apparently more general condition stated for any $x, y$ is actually equivalent. This can be easily seen by considering separately the cases $|x-y| \lesssim t$ and using the size estimate (2.1) and the integral representation of $T$ for $t \lesssim|x-y|$.

Likewise, we say that a bilinear singular integral operator $T: \mathscr{S} \times \mathscr{S} \rightarrow \mathscr{S}^{\prime}$ has the weak boundedness property for bilinear singular integral operators, written $T \in W B P$, if there exists an $M \in \mathbb{N}_{0}$ such that for all normalized bumps $\phi_{1}, \phi_{2}$, and $\phi_{3}$ of order $M, x, y, z \in \mathbb{R}^{n}$ and $t>0$,

$$
\left|\left\langle T\left(\phi_{1}^{x, t}, \phi_{2}^{y, t}\right), \phi_{3}^{z, t}\right\rangle\right| \lesssim t^{n}
$$

Defining the formal transposes of $T$ via

$$
\langle T(f, g), h\rangle=\left\langle T^{* 1}(h, g), f\right\rangle=\left\langle T^{* 2}(f, h), g\right\rangle,
$$

we see that the $W B P$ is symmetric by transposition.

The new tool available that we use to decompose bilinear singular integral operators is the following square function estimate, which is Theorem 1.1 and Remark 2.1 from [16], along with the minor correction in [17.

Theorem 2.4. Suppose $\Theta_{k}$ is defined for $k \in \mathbb{Z}$,

$$
\Theta_{k}(f, g)(x)=\iint \theta_{k}(x, y, z) f(y) g(z) d y d z,
$$

with kernels $\theta_{k}: \mathbb{R}^{n} \times \mathbb{R}^{n} \times \mathbb{R}^{n} \rightarrow \mathbb{C}$ for $k \in \mathbb{Z}$ satisfying

$$
\begin{aligned}
& \left|\theta_{k}(x, y, z)\right| \lesssim \frac{2^{2 k n}}{\left(1+2^{k}|x-y|\right)^{N}\left(1+2^{k}|x-z|\right)^{N}}, \\
& \left|\theta_{k}(x, y, z)-\theta_{k}\left(x, y^{\prime}, z\right)\right| \lesssim 2^{2 k n}\left(2^{k}\left|y-y^{\prime}\right|\right)^{\gamma}, \\
& \left|\theta_{k}(x, y, z)-\theta_{k}\left(x, y, z^{\prime}\right)\right| \lesssim 2^{2 k n}\left(2^{k}\left|z-z^{\prime}\right|\right)^{\gamma}, \\
& \iint \theta_{k}(x, y, z) d y d z=0
\end{aligned}
$$

for some $N>n$ and $0<\gamma \leq 1$. Then for $\frac{1}{p}+\frac{1}{q}=\frac{1}{r}$ for with $1<p, q, r<\infty$,

$$
\left\|\left(\sum_{k \in \mathbb{Z}}\left|\Theta_{k}(f, g)\right|^{2}\right)^{\frac{1}{2}}\right\|_{L^{r}} \lesssim\|f\|_{L^{p}}\|g\|_{L^{q}} .
$$


In fact, in [16] this theorem is proved for a more general accretive setting. Similar results to Theorem 2.4 were proved independently by Grafakos-Oliveira 13 and by Grafakos-Liu-Maldonado-Yang [12 for a certain range of exponents and under a variety of regularity and cancellation assumptions. Here we will need the specific version of Theorem 2.4 as stated above. For previous work on bilinear square functions see for example Maldonado-Naibo [19] and the references therein.

\section{Continuous Decomposition of $T$}

In this section, we shall introduce approximation to the identity operators and Littlewood-Paley projection operators to decompose the operator $T$. We decompose $T$ using operators depending on a continuous parameter $t$ and then translate to the discrete setting of Theorem 2.4. Let $\varphi \in \mathcal{D}$ radial with $\operatorname{supp}(\varphi) \subset B(0,1 / 2)$ and $\widehat{\varphi}(0)=1$, which will be fixed for Sections 3 and 4 . Let $P_{t}$ be defined by $P_{t} f=\varphi_{t} * f$ and let $P_{t}^{2} f=\varphi_{t} * \varphi_{t} * f$. We now prove a proposition to decompose a bilinear singular $T$.

Proposition 3.1. There exist operators $Q_{t}^{1, j}, Q_{t}^{2, j}$ and $t_{j, k} \in\left[2^{-(k+1)}, 2^{-k}\right]$ for $t>0, j=1, \ldots, n$ and $k \in \mathbb{Z}$ such that for all $f, g, h \in \mathscr{S}_{0}$,

$$
\begin{aligned}
\langle T(f, g), h\rangle= & \sum_{j=1}^{n} \sum_{k \in \mathbb{Z}}\left\langle\Theta_{k}^{0, j}(f, g), Q_{t_{j, k}}^{2, j} h\right\rangle+\left\langle\Theta_{k}^{1, j}(h, g), Q_{t_{j, k}}^{2, j} f\right\rangle \\
& +\left\langle\Theta_{k}^{2, j}(f, h), Q_{t_{j, k}}^{2, j} g\right\rangle
\end{aligned}
$$

where

$$
\Theta_{k}^{i, j}(f, g)=\frac{2^{-(k+1)}}{t_{j, k}} Q_{t_{j, k}}^{1, j *} T^{* i}\left(P_{t_{j, k}}^{2} f, P_{t_{j, k}}^{2} g\right)
$$

for $i=0,1,2$ and $j=1,2, \ldots, n$. Furthermore $Q_{t}^{1, j}, Q_{t}^{2, j}$ are convolution operators with kernels $\psi_{t}^{1, j}, \psi_{t}^{2, j} \in \mathcal{D}$ which are supported in $B(0, t)$ and have mean zero.

Proof. First define $Q_{t}^{1, j} f=-2\left(\partial_{x_{j}} \varphi\right)_{t} * f$ and $Q_{t}^{2, j} f=\left(x_{j} \varphi\right)_{t} * f$. Note that the kernels of these operators are smooth functions supported in $B(0, t)$ since $\varphi_{t}$ is smooth with support in $B(0, t)$. Since $P_{t}, Q_{t}^{1, j}$ and $Q_{t}^{2, j}$ are smooth in the parameter $t \in(0, \infty)$, it follows that for $f \in \mathscr{S}$ and $t>0$,

$$
t \frac{d}{d t} P_{t}^{2} f=\sum_{j=1}^{n} Q_{t}^{1, j} Q_{t}^{2, j} f
$$

as a well defined operator that is continuous on $\mathscr{S}$. Recall that for $f \in \mathscr{S}, P_{t}^{2} f \rightarrow f$ as $t \rightarrow 0$ and for $f \in \mathscr{S}_{0}, P_{t}^{2} f \rightarrow 0$ as $t \rightarrow \infty$, where the convergence is in the topology of $\mathscr{S}$. It also follows that for $f, g, h \in \mathscr{S}$ that $P_{t}^{2} f \otimes P_{t}^{2} g \otimes P_{t}^{2} g \rightarrow f \otimes g \otimes h$ as $t \rightarrow 0$ and for $f, g, h \in \mathscr{S}_{0}$ that $P_{t}^{2} f \otimes P_{t}^{2} g \otimes P_{t}^{2} g \rightarrow 0$ as $t \rightarrow \infty$. This uses the fact that $\rho_{\alpha, \beta}\left(P_{t}^{2} f\right)$ is uniformly bounded in $t$ for any Schwartz semi-norm $\rho_{\alpha, \beta}$, 
and similarly for $g$ and $h$. By the continuity of $T$, for any $f, g, h \in \mathscr{S}_{0}$,

$$
\begin{aligned}
\langle T(f, g), h\rangle=\lim _{t \rightarrow 0}\left\langle T\left(P_{t}^{2} f, P_{t}^{2} g\right), P_{t}^{2} h\right\rangle-\lim _{t \rightarrow \infty}\left\langle T\left(P_{t}^{2} f, P_{t}^{2} g\right), P_{t}^{2} h\right\rangle \\
=\int_{0}^{\infty} t \frac{d}{d t}\left\langle P_{t}^{2} T\left(P_{t}^{2} f, P_{t}^{2} g\right), h\right\rangle \frac{d t}{t} \\
=\sum_{j=1}^{n} \sum_{k \in \mathbb{Z}} \int_{2^{-(k+1)}}^{2^{-k}}\left(\left\langle Q_{t}^{1, j} Q_{t}^{2, j} T\left(P_{t}^{2} f, P_{t}^{2} g\right), h\right\rangle\right. \\
+\left\langle P_{t}^{2} T\left(Q_{t}^{1, j} Q_{t}^{2, j} f, P_{t}^{2} g\right), h\right\rangle \\
\left.+\left\langle P_{t}^{2} T\left(P_{t}^{2} f, Q_{t}^{1, j} Q_{t}^{2, j} g\right), h\right\rangle\right) \frac{d t}{t} .
\end{aligned}
$$

Since $P_{t}, Q_{t}^{i, j}$ for $i=0,1,2$ and $j=1,2, \ldots, n$ are continuous in the $t$ parameter in the topology of $\mathscr{S}$ and $T$ is continuous from $\mathscr{S} \times \mathscr{S}$ into $\mathscr{S}^{\prime}$, it follows that $\left\langle T\left(P_{t}^{2} f, P_{t}^{2} g\right), Q_{t}^{1, j} Q_{t}^{2, j} h\right\rangle$ is a continuous function of $t$ for $f, g, h \in \mathscr{S}$. Then by the mean value theorem for integrals for each $j=1,2, \ldots, n$ and $k \in \mathbb{Z}$ there exists $t_{j, k} \in\left[2^{-(k+1)}, 2^{-k}\right]$ such that (3.2) is equal to

$$
\begin{gathered}
\sum_{j=1}^{n} \sum_{k \in \mathbb{Z}}\left(\left\langle Q_{t_{j, k}}^{1, j *} T\left(P_{t_{j, k}}^{2} f, P_{t_{j, k}}^{2} g\right), Q_{t_{j, k}}^{2, j} h\right\rangle+\left\langle Q_{t_{j, k}}^{1, j *} T^{* 1}\left(P_{t_{j, k}}^{2} h, P_{t_{j, k}}^{2} g\right), Q_{t_{j, k}}^{2, j} f\right\rangle\right. \\
\left.+\left\langle Q_{t_{j, k}}^{1, j *} T^{* 2}\left(P_{t_{j, k}}^{2} f, P_{t_{j, k}}^{2} h\right), Q_{t_{j, k}}^{2, j} g\right\rangle\right) \frac{2^{-(k+1)}}{t_{j, k}} \\
=\sum_{k \in \mathbb{Z}} \sum_{j=1}^{n}\left\langle\Theta_{k}^{0, j}(f, g), Q_{t_{j, k}}^{2, j} h\right\rangle+\left\langle\Theta_{k}^{1, j}(h, g), Q_{t_{j, k}}^{2, j} f\right\rangle+\left\langle\Theta_{k}^{2, j}(f, h), Q_{t_{j, k}}^{2, j} g\right\rangle
\end{gathered}
$$

where $\Theta_{k}^{i, j}(f, g)$ is defined for $i=0,1,2$ and $j=1,2, \ldots, n$ as in (3.1). Since $\varphi(x)$ is radial, it follows easily that $x_{j} \varphi(x)$ has mean zero. It is also trivial by the Fundamental Theorem of Calculus that $\partial_{x_{j}} \varphi(x)$ has mean zero. This completes the proof of Proposition 3.1

\section{Reduced T(1) Theorem}

The goal of this section is to prove the reduced $\mathrm{T}(1)$ Theorem, which we state now.

Theorem 4.1. Let $T: \mathscr{S} \times \mathscr{S} \rightarrow \mathscr{S}^{\prime}$ be a bilinear singular integral operator with standard kernel. If $T$ has the weak boundedness property and $T(1,1)=T^{* 1}(1,1)=$ $T^{* 2}(1,1)=0$, then $T$ can be extended to a bounded operator from $L^{p} \times L^{q}$ into $L^{r}$ for all $1<p, q, r<\infty$ with $\frac{1}{p}+\frac{1}{q}=\frac{1}{r}$.

The strategy to prove Theorem 4.1 is to use Proposition 3.1 to decompose $T$, and then use Theorem 2.4 to bound the associated square functions. We will also need a lemma verifying (2.6)-(2.8) for the kernels of $\Theta_{k}^{i, j}$ from Proposition 3.1.

Lemma 4.2. Let $T: \mathscr{S} \times \mathscr{S} \rightarrow \mathscr{S}^{\prime}$ be a bilinear singular integral operator. Let $\varphi_{t}$ be as before and $\psi_{t} \in \mathcal{D}$ have mean zero with $\operatorname{supp}(\psi) \subset B(0,1)$. If $T \in W B P$, $T(1,1)=0$ and $\theta_{k}$ is defined by

$$
\theta_{t}(x, y, z)=\left\langle T\left(\left(\varphi_{t} * \varphi_{t}\right)^{y},\left(\varphi_{t} * \varphi_{t}\right)^{z}\right), \psi_{t}^{x}\right\rangle,
$$


then for some $N>n$,

$$
\begin{aligned}
& \sup _{t \in\left[2^{-(k+1)}, 2^{-k}\right]}\left|\theta_{t}(x, y, z)\right| \lesssim \frac{2^{2 k n}}{\left(1+2^{k}|x-y|\right)^{N}\left(1+2^{k}|x-z|\right)^{N}}, \\
& \sup _{t \in\left[2^{-(k+1)}, 2^{-k}\right]}\left|\theta_{t}(x, y, z)-\theta_{t}\left(x, y^{\prime}, z\right)\right| \lesssim 2^{2 k n}\left(2^{k}\left|y-y^{\prime}\right|\right), \\
& \sup _{t \in\left[2^{-(k+1)}, 2^{-k}\right]}\left|\theta_{t}(x, y, z)-\theta_{t}\left(x, y, z^{\prime}\right)\right| \lesssim 2^{2 k n}\left(2^{k}\left|z-z^{\prime}\right|\right), \\
& \iint \theta_{t}(x, y, z) d y d z=0 \text { for all } t>0 .
\end{aligned}
$$

Proof. To show (4.1), we first assume that $2^{k}(|x-y|+|x-z|+|y-z|) \leq 12$. The weak boundedness of $T$ says that we may test $T$ on normalized bumps of order $M$. Since up to a constant depending on $M, \varphi$ and $\psi$ are normalized bumps of order $M$, for $t \in\left[2^{-(k+1)}, 2^{-k}\right]$

$$
\begin{aligned}
\left|\theta_{t}(x, y, z)\right| & =t^{-3 n}\left|\left\langle T\left((\varphi * \varphi)^{y, t},(\varphi * \varphi)^{z, t}\right), \psi^{x, t}\right\rangle\right| \\
& \lesssim \frac{2^{2 k n}}{\left(1+2^{k}|x-y|\right)^{n+\gamma / 2}\left(1+2^{k}|x-z|\right)^{n+\gamma / 2}} .
\end{aligned}
$$

For $2^{k}(|x-y|+|x-z|+|y-z|)>12$ and $t \in\left[2^{-(k+1)}, 2^{-k}\right]$, assume without loss of generality that $|x-y| \geq \max (|x-z|,|y-z|)$. We would like to use the integral representation of $T$, so we check that $\left(\varphi_{t} * \varphi_{t}\right)^{y}$ and $\psi_{t}^{x}$ have disjoint support. For $v \in \operatorname{supp}\left(\left(\varphi_{t} * \varphi_{t}\right)^{y}\right)$ and $u \in \operatorname{supp}\left(\psi_{t}^{x}\right)$, it follows that $|v-y| \leq t$ and $|u-x| \leq t$. Then for such $u, v$,

$$
|u-v| \geq|x-y|-|u-x|-|v-y| \geq \frac{1}{4}|x-y|+3 \frac{|x-y|}{4}-\geq \frac{1}{4}|x-y|+t .
$$

Hence, using the integral representation of $T$ and that $\psi$ has mean zero,

$$
\begin{aligned}
&\left|\theta_{t}(x, y, z)\right| \\
& \quad=\left|\iiint(K(u, v, w)-K(x, v, w)) \psi_{t}^{x}(u)\left(\varphi_{t} * \varphi_{t}\right)^{y}(v)\left(\varphi_{t} * \varphi_{t}\right)^{z}(w) d u d v d w\right| \\
& \quad \lesssim \int_{|u-x|<t} \int_{|v-y|<t} \int_{|w-z|<t} \frac{t^{-3 n}|u-x|^{\gamma} d w d v d u}{(|u-v|+|u-w|+|v-w|)^{2 n+\gamma}} \\
& \quad \leq \int_{|u-x|<t} \int_{|v-y|<t} \int_{|w-z|<t} \frac{t^{-(3 n-\gamma)} d w d v d u}{(|x-y| / 4+t)^{n+\gamma / 2}(|x-y| / 4+t)^{n+\gamma / 2}} \\
& \quad \lesssim \frac{2^{2 n k}}{\left(1+2^{k}|x-y|\right)^{n+\gamma / 2}\left(1+2^{k}|x-z|\right)^{n+\gamma / 2}} .
\end{aligned}
$$

It follows that $\theta_{t}$ satisfies (4.1). To show (4.2), it is sufficient to note that

$$
\left|\nabla_{y} \theta_{t}(x, y, z)\right|=t^{-1} \sum_{j=1}^{n}\left|\left\langle T\left(\left(\partial_{y_{j}} \varphi * \varphi\right)_{t}^{y},\left(\varphi_{t} * \varphi_{t}\right)^{z}\right), \psi_{t}^{x}\right\rangle\right| \lesssim 2^{k(2 n+1)} .
$$

That same computation holds for (4.3) with $\nabla_{z} \theta_{k}(x, y, z)$. To prove (4.4), note that $\widehat{\varphi * \varphi}(0)=1$ implies that for $R>0$ sufficiently large, $\eta_{R}(x)=\varphi_{t} * \varphi_{t} * \chi_{B(0, R)}(x)$ satisfies $\eta_{R} \in \mathcal{D}$ and $\eta_{R}=1$ on a neighborhood of the origin. So $\eta_{R}$ is an appropriate 
function to compute $T(1,1)$ in the sense of Definition 2.2 . Then it follows that $\Theta_{t}(1,1)=0$ for all $t>0$, as verified in the following computation:

$$
\begin{aligned}
\iint \theta_{t}(x, y, z) d y d z & =\iint\left\langle T\left(\left(\varphi_{t} * \varphi_{t}\right)^{y},\left(\varphi_{t} * \varphi_{t}\right)^{z}\right), \psi_{t}^{x}\right\rangle d y d z \\
& =\lim _{R \rightarrow \infty}\left\langle T\left(\eta_{R}, \eta_{R}\right), \psi_{t}^{x}\right\rangle=0 .
\end{aligned}
$$

The last equality here is given by the assumption $T(1,1)=0$.

The proof of Theorem 4.1 is now easily achieved using Proposition 3.1, Lemma 4.2, and Theorem 2.4.

Proof. Recall the definition of $P_{t}, Q_{t}^{1, j}, Q_{t}^{2, j}$ and $\Theta_{k}^{i, j}$ for $i=0,1,2$ and $j=$ $1,2, \ldots, n$ from Proposition 3.1 For $f, g, h \in \mathscr{S}_{0}$, we apply Proposition 3.1 to get

$$
\begin{aligned}
|\langle T(f, g), h\rangle| \leq \sum_{j=1}^{n} & \left\|\left(\sum_{k \in \mathbb{Z}}\left|\Theta_{k}^{0, j}(f, g)\right|^{2}\right)^{\frac{1}{2}}\right\|\left\|_{L^{r}}\right\|\left(\sum_{k \in \mathbb{Z}}\left|Q_{t_{j, k}}^{2, j} h\right|^{2}\right)^{\frac{1}{2}} \|_{L^{r^{\prime}}} \\
& +\left.\left.\sum_{j=1}^{n}\left\|\left(\sum_{k \in \mathbb{Z}}\left|\Theta_{k}^{1, j}(h, g)\right|^{2}\right)^{\frac{1}{2}}\right\|\right|_{L^{p^{\prime}}}\left\|\left(\sum_{k \in \mathbb{Z}}\left|Q_{t_{j, k}}^{2, j} f\right|^{2}\right)^{\frac{1}{2}}\right\|\left\|_{L^{p}}\right\|\right|_{j=1} ^{\frac{1}{2}}\|\|_{k \in \mathbb{Z}}\left\|\left(\sum_{k \in \mathbb{Z}}\left|Q_{t_{j, k}, j}^{2, j}\right|^{2}\right)^{\frac{1}{2}}\right\| \|_{L^{q}} .
\end{aligned}
$$

The kernels of $\Theta_{k}^{i, j}$ are given by

$$
\theta_{k}^{i, j}(x, y, z)=\left\langle T^{* i}\left(\left(\varphi_{t_{j, k}} * \varphi_{t_{j, k}}\right)^{y},\left(\varphi_{t_{j, k}} * \varphi_{t_{j, k}}\right)^{z}\right), \psi_{t_{j, k}}^{x}\right\rangle
$$

where $\varphi_{t_{j, k}}(x-y)$ is the kernel of $P_{t_{j, k}}$ and $\psi_{t_{j, k}}(x-y)$ is the kernel of $Q_{t}^{1, j *}$. Then for each $i=0,1,2$ and $j=1,2, \ldots, n$ we have that $\varphi_{t} * \varphi_{t}$ and $\psi_{t}$ satisfy the hypotheses of Lemma 4.2. Hence $\varphi_{t_{j, k}} * \varphi_{t_{j, k}}, \psi_{t_{j, k}}$ and $\Theta_{k}^{i, j}$ satisfy (2.6)(2.9). Then by Theorem 2.4 it follows that $\Theta_{k}^{i, j}$ satisfies (2.10) for $i=0,1,2$ and $j=1,2, \ldots, n$. Then applying this bound with $\frac{1}{p}+\frac{1}{q}=\frac{1}{r}$ for $\Theta_{k}^{0, j}, \frac{1}{r^{\prime}}+\frac{1}{q}=\frac{1}{p^{\prime}}$ for $\Theta_{k}^{1, j}$ and $\frac{1}{p}+\frac{1}{r^{\prime}}=\frac{1}{q^{\prime}}$ for $\Theta_{k}^{2, j}$, it follows that

$$
|\langle T(f, g), h\rangle| \lesssim\|f\|_{L^{p}}\|g\|_{L^{q}}\|h\|_{L^{r^{\prime}}}+\|h\|_{L^{r^{\prime}}}\|g\|_{L^{q}}\|f\|_{L^{p}}+\|f\|_{L^{p}}\|h\|_{L^{r^{\prime}}}\|g\|_{L^{q}} .
$$

Note that it is important here that $1<p, q, r<\infty$ so that $1<p^{\prime}, q^{\prime}, r^{\prime}<\infty$, and we can apply Theorem 2.4 for $1<p, q, r, p^{\prime}, q^{\prime}, r^{\prime}<\infty$ and Littlewood-Paley estimates for $1<p, q, r^{\prime}<\infty$. Since $\mathscr{S}_{0}$ is dense in $L^{p}$ for $1<p<\infty$, this completes the proof of Lemma 4.2 .

\section{A paraproduct construction and the full T(1) Theorem}

In this section we construct paraproducts that allow the reduction of Theorem 1.1 to Theorem 4.1 .

Lemma 5.1. Given $\beta \in B M O$, there exists a bilinear Calderón-Zygmund operator $L$ bounded on $L^{p} \times L^{q} \rightarrow L^{r}$ for all $1<p, q<\infty$ with $\frac{1}{p}+\frac{1}{q}=\frac{1}{r}$, satisfying

$$
L(1,1)=\beta \text { and } L^{* 1}(1,1)=L^{* 2}(1,1)=0 .
$$


Proof. Let $P_{t} f=\varphi_{t} * f$ be a smooth approximation to the identity for some $\varphi \in \mathcal{D}$ that is radial with $\operatorname{supp}(\varphi) \subset B(0,1)$. Also $Q_{t} f=\psi_{t} * f$, where $\psi$ is a radial compactly supported function with mean zero, and

$$
\int_{0}^{\infty} \widehat{\psi}\left(t e_{1}\right)^{2} \frac{d t}{t}=1,
$$

where $e_{1}=(1,0, \ldots, 0) \in \mathbb{R}^{n}$. Then it follows that

$$
\int_{0}^{\infty} Q_{t}^{2} f \frac{d t}{t}=f
$$

in $L^{p}$ for all $1<p<\infty$ and in $H^{1}$. This is a classical result in $L^{p}$ for $1<p<\infty$. For $H^{1}$, this fact was originally due to Folland-Stein [8] in the discrete setting and to Wilson in 23] in the continuous setting as used here. Define $L$ with kernel $\ell(x, y, z)$ by the following:

$$
\begin{aligned}
L(f, g) & =\int_{0}^{\infty} L_{t}(f, g) \frac{d t}{t}=\int_{0}^{\infty} Q_{t}\left(\left(Q_{t} \beta\right)\left(P_{t} f P_{t} g\right)\right) \frac{d t}{t} \\
\ell(x, y, z) & =\int_{0}^{\infty} \ell_{t}(x, y, z) \frac{d t}{t}=\int_{0}^{\infty} \int \psi_{t}(x-u) Q_{t} \beta(u) \varphi_{t}(u-y) \varphi_{t}(u-z) d u \frac{d t}{t} .
\end{aligned}
$$

Define the non-negative measure $d \mu$ on $\mathbb{R}_{+}^{n+1}$ by

$$
d \mu(x, t)=\left|Q_{t} \beta(x)\right|^{2} d x \frac{d t}{t} .
$$

We should first note that $Q_{t} \beta$ is well defined since $Q_{t} 1=0$. Further, $Q_{t}$ are Littlewood-Paley-Stein projection operators, so the square function associated to $Q_{t}$ is bounded on $L^{p}$ for $1<p<\infty$. It also follows that (5.1) is a Carleson measure. This type of result was originally proved by Carleson in [4 and further developed by Jones in [18. A proof of this result can be found as Theorem 7.3.8(c) in 11. Then for any $f, g, h \in \mathscr{S}$ with $\|h\|_{L^{2}} \leq 1$, we have for $1<p, q<\infty$ such that $\frac{1}{p}+\frac{1}{q}=\frac{1}{2}$ :

$$
\begin{aligned}
&|\langle L(f, g), h\rangle| \leq \int_{0}^{\infty}\left|\int\left(Q_{t} \beta(x) P_{t} f(x) P_{t} g(x)\right) Q_{t} h(x) d x\right| \frac{d t}{t} \\
& \leq\left.\left(\int_{0}^{\infty} \int\left|P_{t} f(x)\right|^{2}\left|P_{t} g(x)\right|^{2}\left|Q_{t} \beta(x)\right|^{2} d x \frac{d t}{t}\right)^{\frac{1}{2}}\left\|\left(\int_{0}^{\infty}\left|Q_{t} h\right|^{2} \frac{d t}{t}\right)^{\frac{1}{2}}\right\|\right|_{L^{2}} \\
& \lesssim\left(\int_{0}^{\infty} \int\left|P_{t} f(x)\right|^{p}\left|Q_{t} \beta(x)\right|^{2} d x \frac{d t}{t}\right)^{\frac{1}{p}} \\
& \times\left(\int_{0}^{\infty} \int\left|P_{t} g(x)\right|^{q}\left|Q_{t} \beta(x)\right|^{2} d x \frac{d t}{t}\right)^{\frac{1}{q}}\|h\|_{L^{2}} \\
& \lesssim\|f\|_{L^{p}}\|g\|_{L^{q}} .
\end{aligned}
$$

In the last inequality, we use a result also due to Carleson. A proof of this estimate can be found in [1], stated as Theorem 7.3.7. So we have proved that $L$ is bounded from $L^{p} \times L^{q}$ into $L^{2}$ for any $1<p, q<\infty$ with $\frac{1}{p}+\frac{1}{q}=\frac{1}{2}$. We now show that 
$\ell(x, y, z)$ satisfies (2.1)-(2.4) as well. To see that $\ell$ satisfies (2.1), first consider $\ell_{t}$ :

$$
\begin{aligned}
\left|\ell_{t}(x, y, z)\right| \leq & || Q_{t} \beta \|_{L^{\infty}} \int\left|\psi_{t}(x-u) \varphi_{t}(u-y) \varphi_{t}(u-z)\right| d u \\
\lesssim & \int \frac{t^{-n}}{\left(1+t^{-1}|x-u|\right)^{4(n+1)}} \\
& \times \frac{t^{-2 n}}{\left(1+t^{-1}|u-y|\right)^{4(n+1)}\left(1+t^{-1}|u-z|\right)^{4(n+1)}} d u \\
\lesssim & \frac{t^{-2 n}}{\left(1+t^{-1} \max (|x-y|,|x-z|)\right)^{4(n+1)}} \\
\leq & \frac{t^{-2 n}}{\left(1+t^{-1}|x-y|\right)^{2(n+1)}\left(1+t^{-1}|x-z|\right)^{2(n+1)}} .
\end{aligned}
$$

Note that here we use $\left\|Q_{t} \beta\right\|_{L^{\infty}} \lesssim\|\beta\|_{B M O}$. Similarly for (2.2) we have

$$
\begin{aligned}
\mid \ell_{t}(x, y & , z)-\ell_{t}\left(x^{\prime}, y, z\right) \mid \\
& \leq \int\left|\psi_{t}(x-u)-\psi_{t}\left(x^{\prime}-u\right)\right|\left|Q_{t} \beta(u) \varphi(u-y) \varphi(u-z)\right| d u \\
& \lesssim \int \frac{t^{-n}\left(t^{-1}\left|x-x^{\prime}\right|\right)}{\left(1+t^{-1}|x-u|\right)^{4(n+1)}} \frac{t^{-2 n}}{\left(1+t^{-1}|u-y|\right)^{4(n+1)}\left(1+t^{-1}|u-z|\right)^{4(n+1)}} d u \\
& \lesssim \frac{t^{-2 n}\left(t^{-1}\left|x-x^{\prime}\right|\right)}{\left(1+t^{-1}|x-y|\right)^{2(n+1)}\left(1+t^{-1}|x-z|\right)^{2(n+1)}} .
\end{aligned}
$$

It then follows by taking $d=|x-y|+|x-z|$ that

$$
|\ell(x, y, z)| \leq d^{-2(n+1)} \int_{0}^{d} \frac{t^{-2 n}}{t^{-2(n+1)}} \frac{d t}{t}+\int_{d}^{\infty} t^{-2 n} \frac{d t}{t} \lesssim d^{-2 n} .
$$

Similarly we have

$$
\begin{aligned}
\left|\ell(x, y, z)-\ell\left(x^{\prime}, y, z\right)\right| & \lesssim\left|x-x^{\prime}\right| d^{-2(n+1)} \int_{0}^{d} \frac{t^{-(2 n+1)}}{t^{-2(n+1)}} \frac{d t}{t}+\left|x-x^{\prime}\right| \int_{d}^{\infty} t^{-(2 n+1)} \frac{d t}{t} \\
& \lesssim\left|x-x^{\prime}\right| d^{-(2 n+1)} .
\end{aligned}
$$

With symmetric arguments for (2.3) and (2.4), the kernel $\ell$ satisfies (2.1)-(2.4). So $L$ is a Calderón-Zygmund operator and is bounded on $L^{p} \times L^{q} \rightarrow L^{r}$ for all $1<$ $p, q<\infty$ where $\frac{1}{p}+\frac{1}{q}=\frac{1}{r}$. Let $\eta \in \mathcal{D}$ with $\eta \equiv 1$ on $B(0,1)$ and $\operatorname{supp}(\eta) \subset B(0,2)$. Let $\phi \in \mathcal{D}$ with mean zero and $N$ such that $\operatorname{supp}(\phi) \subset B(0, N)$. Then to compute $L(1,1)$ in the sense of Definition 2.2

$$
\begin{aligned}
\langle L(1,1), \phi\rangle=\lim _{R \rightarrow \infty} \int_{0}^{R / 4} & \int Q_{t} \phi(x)\left[P_{t} \eta_{R}(x)\right]^{2} Q_{t} \beta(x) d x \frac{d t}{t} \\
& +\lim _{R \rightarrow \infty} \int_{R / 4}^{\infty} \int Q_{t} \phi(x)\left[P_{t} \eta_{R}(x)\right]^{2} Q_{t} \beta(x) d x \frac{d t}{t} .
\end{aligned}
$$

We may write this only if the two limits on the right hand side of the equation exist. As we are taking $R \rightarrow \infty$ and $N$ is a fixed quantity determined by $\phi$, without loss of generality assume that $R>2 N$. Note that for $t \leq R / 4$ and $|x|<N+t$,

$$
\operatorname{supp}\left(\varphi_{t}(x-\cdot)\right) \subset B(x, t) \subset B(0, N+2 t) \subset B(0, R) .
$$


Since $\eta_{R} \equiv 1$ on $B(0, R)$, it follows that $P_{t} \eta_{R}(x)=1$ for all $|x|<N+t$ when $t \leq R / 4$. Therefore

$$
\lim _{R \rightarrow \infty} \int_{0}^{R / 4} \int Q_{t} \phi(x)\left[P_{t} \eta_{R}(x)\right]^{2} Q_{t} \beta(x) d x \frac{d t}{t}=\int_{0}^{\infty} \int Q_{t}^{2} \phi(x) \beta(x) d x \frac{d t}{t}=\langle\beta, \phi\rangle,
$$

where we have used that Calderón's reproducing formula holds in $H^{1}$. For any $t>0$

$$
\begin{aligned}
& \left\|P_{t} \eta_{R}\right\|_{L^{1}} \lesssim\left\|\varphi_{t} \mid\right\|_{L^{1}}\left\|\eta_{R}\right\|_{L^{1}} \lesssim R^{n} \\
& \left\|P_{t} \eta_{R}\right\|_{L^{\infty}} \leq\left\|\varphi_{t}\right\|_{L^{1}}\left\|\eta_{R}\right\|_{L^{\infty}}=1
\end{aligned}
$$

and for any $x \in \mathbb{R}^{n}$,

$$
\left|Q_{t} \phi(x)\right|=\left|\int\left(\psi_{t}(x-y)-\psi_{t}(x)\right) \phi(y) d y\right| \lesssim t^{-(n+1)} .
$$

Therefore

$$
\begin{aligned}
\int_{R / 4}^{\infty} & \int\left|Q_{t} \phi(x)\left[P_{t} \eta_{R}(x)\right]^{2} Q_{t} \beta(x)\right| d x \frac{d t}{t} \\
& \leq \int_{R / 4}^{\infty}\left\|P_{t} \eta_{R}\right\|_{L^{1}}\left\|P_{t} \eta_{R}\right\|_{L^{\infty}}\left\|Q_{t} \beta\right\|_{L^{\infty}}\left\|Q_{t} \phi\right\|_{L^{\infty}} \frac{d t}{t} \\
& \lesssim \int_{R / 4}^{\infty} R^{n} t^{-(n+1)} \frac{d t}{t} \lesssim R^{-1} .
\end{aligned}
$$

Hence the second limit in (5.2) exists and tends to 0 as $R \rightarrow \infty$. Then $\langle L(1,1), \phi\rangle=$ $\langle\beta, \phi\rangle$ for all $\phi \in \mathcal{D}$ with mean zero and hence $L(1,1)=\beta$ as an element of BMO. Again for any $\phi \in \mathcal{D}$ with mean zero and $\operatorname{supp}(\phi) \subset B(0, N)$, we have

$$
\begin{aligned}
\left\langle L^{* 1}(1,1), \phi\right\rangle= & \lim _{R \rightarrow \infty} \int_{0}^{R / 4} \int_{|x|<N+t} Q_{t} \beta(x) P_{t} \phi(x) P_{t} \eta_{R}(x) Q_{t} \eta_{R}(x) d x \frac{d t}{t} \\
& +\lim _{R \rightarrow \infty} \int_{R / 4}^{\infty} \int_{|x|<N+t} Q_{t} \beta(x) P_{t} \phi(x) P_{t} \eta_{R}(x) Q_{t} \eta_{R}(x) d x \frac{d t}{t} .
\end{aligned}
$$

Once more, without loss of generality take $R>2 N$. When $|x|<N+t$ and $t \leq R / 4$

$$
\operatorname{supp}\left(\psi_{t}(x-\cdot)\right) \subset B(x, t) \subset B(0, N+2 t) \subset B(0, R),
$$

and hence $Q_{t} \eta_{R}(x)=Q_{t} 1(x)=0$. With this it is apparent that the first limit in (5.7) is 0. Similar to (5.3)-(5.5), for the terms of (5.7) we have $\left\|P_{t} \eta_{R}\right\|_{L^{1}} \lesssim R^{n}$, $\left\|Q_{t} \eta_{R}\right\|_{L^{\infty}} \lesssim 1$, and $\left\|P_{t} \phi\right\|_{L^{\infty}} \lesssim t^{-(n+1)}$. So the second term of (5.7) tends to 0 as $R \rightarrow \infty$, just like the second term in computing $L(1,1)$ from (5.6). Then $L^{* 1}(1,1)=0$, and a similar argument proves that $L^{* 2}(1,1)=0$, which concludes the proof of Lemma 5.1 .

Finally we prove the full bilinear T(1) Theorem, that is, Theorem 1.1 .

Proof. By Lemma 5.1, there exist bilinear Calderón-Zygmund operators $L_{0}, L_{1}, L_{2}$ such that $L_{i}^{* i}(1,1)=T^{* i}(1,1)$ and $L_{j}^{* i}(1,1)=0$ for $i \neq j$. Define $S: \mathscr{S} \times \mathscr{S} \rightarrow \mathscr{S}^{\prime}$ by $S(f, g)=T(f, g)-L_{0}(f, g)-L_{1}(f, g)-L_{2}(f, g)$, which by Theorem 4.1 is bounded from $L^{p} \times L^{q}$ into $L^{r}$ for all $1<p, q, r<\infty$ with $\frac{1}{p}+\frac{1}{q}=\frac{1}{r}$. Therefore $T$ is bounded on the same spaces. It follows from a bilinear Calderón-Zygmund weak type endpoint estimate and interpolation that $T$ is bounded from $L^{p} \times L^{q}$ into $L^{r}$ for all $1<p, q<\infty$ such that $\frac{1}{p}+\frac{1}{q}=\frac{1}{r}$ (see for example [14] or [15]). 
The necessity of the conditions in the theorem are by now also well known; see for example [15].

We conclude by observing that our version of the $T(1)$ Theorem still easily applies to one of the main applications of the result. Namely it applies to bilinear pseudodifferential operators

$$
T(f, g)(x)=\int p(x, \xi, \eta) \widehat{f}(\xi) \widehat{g}(\eta) e^{i x(\xi+\eta)} d \xi d \eta
$$

with symbols in the classes $B S_{1, \delta}^{0}, 0 \leq \delta<1$. These operators have standard kernels, and the classes $B S_{1, \delta}^{0}$ are closed under transposition for $\delta<1$. Such an operator trivially satisfies the $W B P$ with normalized bumps of order $M=2[n / 2+1]$ where $[x]$ is the integer part of $x$,

$$
\begin{aligned}
\left|\left\langle T\left(\phi_{1}^{x, t}, \phi_{2}^{y, t}\right), \phi_{3}^{z, t}\right\rangle\right| & \leq\|p\|_{L^{\infty}}\left\|\widehat{\phi_{1}^{x, t}}\right\|_{L^{1}}\left\|\widehat{\phi_{2}^{y, t}}\right\|_{L^{1}}\left\|\phi_{3}^{z, t}\right\|_{L^{1}} \\
& \lesssim t^{n}\|p\|_{L^{\infty}} \sum_{j=0}^{M / 2} \sum_{k=0}^{M / 2}\left\|\Delta^{j} \phi_{1}\right\|_{L^{\infty}}\left\|\Delta^{k} \phi_{2}\right\|_{L^{\infty}}\left\|\phi_{3}\right\|_{L^{\infty}} .
\end{aligned}
$$

Formally we have that $T(1,1)=p(\cdot, 0,0) \in L^{\infty} \subset B M O$, and similarly for the transposes of $T$. Then boundedness of $T$ follows from Theorem 1.1. See 3 for further definitions, details and other related works.

\section{ACKNowledGements}

This work is part of the author's Ph.D. dissertation in the mathematics department at the University of Kansas. The author would like to express his sincere gratitude to Rodolfo $\mathrm{H}$. Torres for his guidance and support throughout this work. The author would also like to thank the anonymous referees for their suggestions and comments.

\section{REFERENCES}

[1] Árpád Bényi, Bilinear singular integral operators, smooth atoms and molecules, J. Fourier Anal. Appl. 9 (2003), no. 3, 301-319, DOI 10.1007/s00041-003-0016-y. MR.1988754 (2004c:42028)

[2] Árpád Bényi, Ciprian Demeter, Andrea R. Nahmod, Christoph M. Thiele, Rodolfo H. Torres, and Paco Villarroya, Modulation invariant bilinear T(1) theorem, J. Anal. Math. 109 (2009), 279-352, DOI 10.1007/s11854-009-0034-z. MR2585397(2011b:42032)

[3] Árpád Bényi, Diego Maldonado, Virginia Naibo, and Rodolfo H. Torres, On the Hörmander classes of bilinear pseudodifferential operators, Integral Equations Operator Theory 67 (2010), no. 3, 341-364, DOI 10.1007/s00020-010-1782-y. MR2660466 (2011g:47111)

[4] Lennart Carleson, An interpolation problem for bounded analytic functions, Amer. J. Math. 80 (1958), 921-930. MR0117349 (22 \#8129)

[5] Ronald R. Coifman and Yves F. Meyer, A simple proof of a theorem by G. David and J.-L. Journé on singular integral operators, Probability theory and harmonic analysis (Cleveland, Ohio, 1983), Monogr. Textbooks Pure Appl. Math., vol. 98, Dekker, New York, 1986, pp. 6165. MR830231 (87f:47075)

[6] Michael Christ and Jean-Lin Journé, Polynomial growth estimates for multilinear singular integral operators, Acta Math. 159 (1987), no. 1-2, 51-80, DOI 10.1007/BF02392554. MR.906525 (89a:42024)

[7] Guy David and Jean-Lin Journé, A boundedness criterion for generalized Calderón-Zygmund operators, Ann. of Math. (2) 120 (1984), no. 2, 371-397, DOI 10.2307/2006946. MR763911 (85k:42041) 
[8] G. B. Folland and Elias M. Stein, Hardy spaces on homogeneous groups, Mathematical Notes, vol. 28, Princeton University Press, Princeton, N.J.; University of Tokyo Press, Tokyo, 1982. MR.657581 (84h:43027)

[9] M. Frazier, Y.-S. Han, B. Jawerth, and G. Weiss, The T1 theorem for Triebel-Lizorkin spaces, Harmonic analysis and partial differential equations (El Escorial, 1987), Lecture Notes in Math., vol. 1384, Springer, Berlin, 1989, pp. 168-181, DOI 10.1007/BFb0086801. MR1013823 (90j:46035)

[10] M. Frazier, R. Torres, and G. Weiss, The boundedness of Calderón-Zygmund operators on the spaces $\dot{F}_{p}^{\alpha, q}$, Rev. Mat. Iberoamericana 4 (1988), no. 1, 41-72, DOI 10.4171/RMI/63. MR:1009119 (90k:42029)

[11] Loukas Grafakos, Modern Fourier analysis, 2nd ed., Graduate Texts in Mathematics, vol. 250, Springer, New York, 2009. MR2463316 (2011d:42001)

[12] L. Grafakos, L. Liu, D. Maldonado, and D. Yang, Multilinear analysis on metric spaces, Dissertationes Math. (Rozprawy Mat.) 497 (2014), 121 pp. MR.3183648

[13] L. Grafakos and L. Oliveira, Carleson measures associated with families of multilinear operators, Studia Math. 211 (2012), no. 1, 71-94. MR2990559

[14] Loukas Grafakos and Rodolfo H. Torres, On multilinear singular integrals of CalderónZygmund type, Proceedings of the 6th International Conference on Harmonic Analysis and Partial Differential Equations (El Escorial, 2000), 2002, pp. 57-91, DOI 10.5565/PUBLMAT_Esco02_04. MR1964816 (2004c:42031)

[15] Loukas Grafakos and Rodolfo H. Torres, Multilinear Calderón-Zygmund theory, Adv. Math. 165 (2002), no. 1, 124-164, DOI 10.1006/aima.2001.2028. MR1880324 (2002j:42029)

[16] J. Hart, Bilinear square functions and vector-valued Calderón-Zygmund operators, J. Fourier Anal. Appl. 18 (2012), no. 6, 1291-1313. MR3000983

[17] J. Hart, Erratum to: "Square functions and vector-valued Calderón-Zygmund operators", J. Fourier Anal. Appl. 20 (2014), no. 1, 222-224. MR3180895

[18] Peter W. Jones, Square functions, Cauchy integrals, analytic capacity, and harmonic measure, Harmonic analysis and partial differential equations (El Escorial, 1987), Lecture Notes in Math., vol. 1384, Springer, Berlin, 1989, pp. 24-68, DOI 10.1007/BFb0086793. MR1013815 (91b:42032)

[19] Diego Maldonado and Virginia Naibo, On the boundedness of bilinear operators on products of Besov and Lebesgue spaces, J. Math. Anal. Appl. 352 (2009), no. 2, 591-603, DOI 10.1016/j.jmaa.2008.11.020. MR2501903(2009m:42024)

[20] Yves Meyer, Les nouveaux opérateurs de Calderón-Zygmund (French), Astérisque 131 (1985), 237-254. Colloquium in honor of Laurent Schwartz, Vol. 1 (Palaiseau, 1983). MR.816750 (87f:42040b)

[21] Elias M. Stein, Harmonic analysis: real-variable methods, orthogonality, and oscillatory integrals, with the assistance of Timothy S. Murphy; Monographs in Harmonic Analysis, III. Princeton Mathematical Series, vol. 43, Princeton University Press, Princeton, NJ, 1993. MR,1232192(95c:42002)

[22] Rodolfo H. Torres, Boundedness results for operators with singular kernels on distribution spaces, Mem. Amer. Math. Soc. 90 (1991), no. 442, viii+172. MR1048075 (91g:47044)

[23] Michael Wilson, Convergence and stability of the Calderón reproducing formula in $H^{1}$ and $B M O$, J. Fourier Anal. Appl. 17 (2011), no. 5, 801-820, DOI 10.1007/s00041-010-9165-y. $\operatorname{MR} 2838108$

Department of Mathematics, University of Kansas, LaWrence, Kansas 66045

E-mail address: jhart@math.ku.edu 